\title{
A Novel Spirocyclic Halogen-Free Flame Retardant: Synthesis and Flammability Characteristics in Epoxy Resin Composites
}

\author{
Hongcheng $\mathrm{Li}^{\mathrm{a}}$, Jiping $\mathrm{Liu}^{\mathrm{b}}{ }^{*}$ and Jiayi Zhao ${ }^{\mathrm{c}}$
}

School of Materials Science \& Engineering, Beijing Institute of Technology, Beijing 100081, China

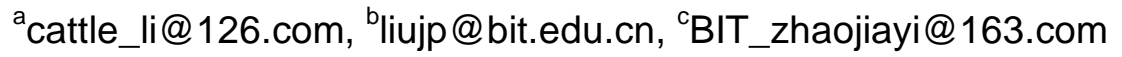

Keywords: Intumescent flame retardant; Imidazole spirocyclic phosphoramidate; Epoxy resin Abstract. A novel spirocyclic halogen-free flame retardant ISCP was successfully synthesized, and its structure was confirmed by FTIR, ${ }^{1} \mathrm{H}$ NMR, and ${ }^{31} \mathrm{P}$ NMR. Results of LOI, UL-94, and CONE tests demonstrated that the ISCP effectively enhanced flame retardancy of EP composites, which could improve the LOI value from 19.5 to 36.8 , pass the UL-94 V-0 rating when $20 \mathrm{wt}$.\% ISCP was added, and CONE tests suggested that ISCP could decrease the PHRR and THR remarkably. TGA tests revealed that during the combustion, ISCP could increase the amount of residual char. SEM morphologies indicated that ISCP greatly facilitated the char-forming process and generated uniform and compact intumescent char layer, leading to EP with improved flame retardancy.

\section{Introduction}

Epoxy resin (EP) has been one of the most widely applied materials in electronic industry due to their superior electric insulation performance and high temperature resistance [1,2]. However, its application is greatly limited due to the fateful drawbacks as we known the high flammability, emission of smoke and poisonous gas in burning process [3]. The most promising approaches to overcome above defects of EP involve the utilization of phosphate flame retardants. Among these flame retardants, the spirocyclic phosphate has high thermal stability because of its rigid spiro structure, which is a stable six-member heterocycle consisted of $\mathrm{P}, \mathrm{O}, \mathrm{C}$. Meanwhile, pentaerythritol charring effect of the spirocyclic phosphate contributes to fully exerting both gaseous- and condensed-phase effects, which could inhibit further combustion [4].

In this study, a novel spirocyclic halogen-free flame retardant, imidazole spirocyclic phosphoramidate (ISCP) has been successfully synthesized by condensation reaction of imidazole with SPDPC. Moreover, ISCP was blended with EP to improve its fire performance. Flame retardancy and thermal decomposition behavior of the EP composites have been investigated.

\section{Experimental}

Materials. Imidazole, triethylamine, chloroform, acetone, dichloromethane, ethanediamine and pentaerythritol (PER) were supplied by Beijing Chemical Agent Corporation. Phosphorus oxychloride $\left(\mathrm{POCl}_{3}\right)$ was purchased from Tingxin Chemical Industry Corporation. EP (DGEBA, commercial name: E-44) was purchased from Sinopec Baling Company.

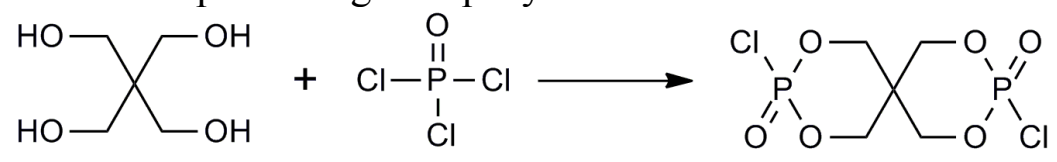

SPDPC

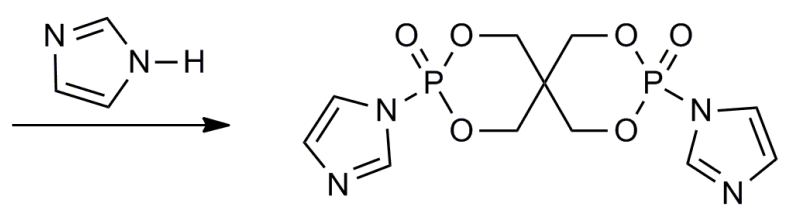

ISCP

Scheme 1 Synthesis equation and structure of ISCP. 
Synthesis of ISCP. As shown in Scheme 1, the intermediate SPDPC was synthesized by esterification reaction of PER and $\mathrm{POCl}_{3}$ according to previous literature [5]. Afterwards, dichloromethane (50 $\left.\mathrm{mL}\right)$, imidazole (1.64 g, $0.024 \mathrm{~mol})$ and SPDPC $(2.97 \mathrm{~g}, 0.01 \mathrm{~mol})$ were mixed in a $250 \mathrm{~mL}$ three-neck flask equipped with a mechanical stirrer, a thermometer, a reflux condenser and $\mathrm{HCl}$ absorption device. Then triethylamine $(2.8 \mathrm{~mL})$ was added gradually by drops at room temperature. Thereafter, the mixture was gradually heated to $60{ }^{\circ} \mathrm{C}$ and maintained $6 \mathrm{~h}$. It was then cooled to room temperature. The raw product obtained was filtered and purified by chloroform and acetone sequentially. The target product was dried to constant weight at $60{ }^{\circ} \mathrm{C}$ in vacumm to get white solid powder (yield: $73.6 \%$ ).

Preparation of EP/ISCP Composites. EP and ISCP were dried under vacumm at $60{ }^{\circ} \mathrm{C}$ for $6 \mathrm{~h}$. Then EP and ISCP were blended into a $250 \mathrm{~mL}$ three-neck flask equipped with mechanical stirrer and a thermometer according to the formulas presented in Table 1, and stirred until liquid tended to be homogeneous. Thereafter the curing agent ethanediamine was added and stirred. The composites were poured into molds, which cured at $80{ }^{\circ} \mathrm{C}$ for $2 \mathrm{~h}$ and post-cured at $120{ }^{\circ} \mathrm{C}$ for the succeeding $2 \mathrm{~h}$, and then cooled to room temperature to obtain the composite specimens.

Measurements and Characterization. Fourier transform infrared (FTIR) spectrum was recorded by a Bruker TENSOR 27 spectrophotometer with $\mathrm{KBr}$ pellets in the 500 4000 $\mathrm{cm}^{-1}$ region. Nuclear magnetic resonance (NMR) was performed on a Bruker $400(400-\mathrm{MHz})$ NMR spectrometer with $d_{6}$-DMSO as a solvent. Limiting oxygen index (LOI) was carried out using an HC-2 type instrument on the specimens of $120 \times 6.5 \times 3 \mathrm{~mm}^{3}$ according to ASTM D2863. The UL-94 test was performed by a CZF-3 type instrument on the specimens of $127 \times 12.7 \times 3 \mathrm{~mm}^{3}$ according to ASTM D635. CONE measurements were made by an FTT0007 CONE calorimeter according to ISO 5660 under an external heat flux of $50 \mathrm{~kW} / \mathrm{m}^{2}$. Thermogravimetric analysis (TGA) was measured using a METTLER TOLEDO STARe apparatus. Measurements were performed at a heating rate of $20{ }^{\circ} \mathrm{C} / \mathrm{min}$ under a nitrogen flow of $20 \mathrm{~mL} / \mathrm{min}$. Scanning electron microscopy (SEM) analyses for the morphology of the residual char after combustion by CONE tests were performed with a Hitachi S-4800 scanning electron microscopy. The gold-coated specimens to avoid accumulation of charges were analyzed at an accelerating voltage of $5 \mathrm{kV}$.

\section{Results and Discussion}

Characterization of ISCP. Fig. 1 shows FTIR spectrum of the synthesized ISCP. The characteristic bands at $2966 \mathrm{~cm}^{-1}(\mathrm{C}-\mathrm{H}), 1313 \mathrm{~cm}^{-1}(\mathrm{P}=\mathrm{O}), 1157 \mathrm{~cm}^{-1}(\mathrm{P}-\mathrm{O}-\mathrm{C})$ were observed. Meanwhile the consumption of the $\mathrm{P}-\mathrm{Cl}$ group was observed through the disappearance of the characteristic bands at $540 \sim 560 \mathrm{~cm}^{-1}$.

To further confirm the structure of ISCP, NMR measurements were employed. Fig. 2 shows the ${ }^{1} \mathrm{H}$ NMR and ${ }^{31} \mathrm{P}$ NMR spectra of ISCP. It can be seen that the the characteristic peaks of ISCP at 3.95 and $3.98 \mathrm{ppm}\left(-\mathrm{CH}_{2^{-}}\right.$, labeled a), $7.54 \mathrm{ppm}(\mathrm{C}-\mathrm{CH}-\mathrm{N}$, labeled b), $8.82 \mathrm{ppm}(\mathrm{N}-\mathrm{CH}-\mathrm{N}$, labeled c). The signal at -5.11 was ascribed to phosphorus atoms which had the same chemical environment in ISCP. From the above analysis, the chemical structure of ISCP was determined.

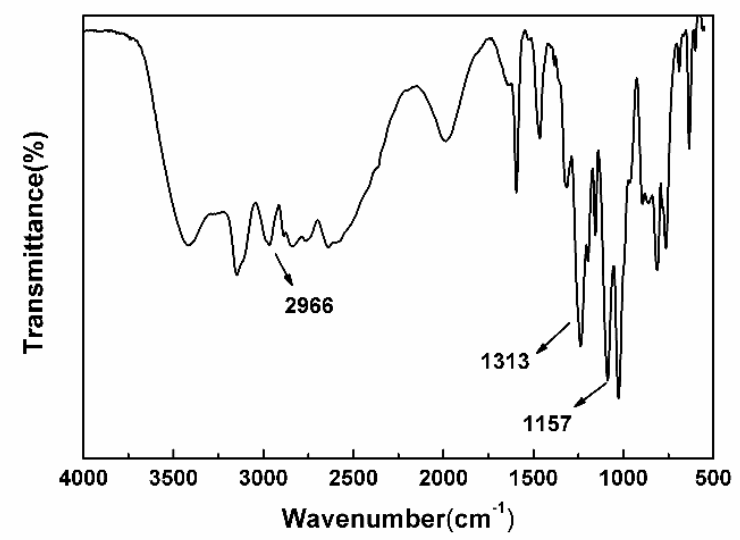

Fig. 1 FTIR spectrum of ISCP. 

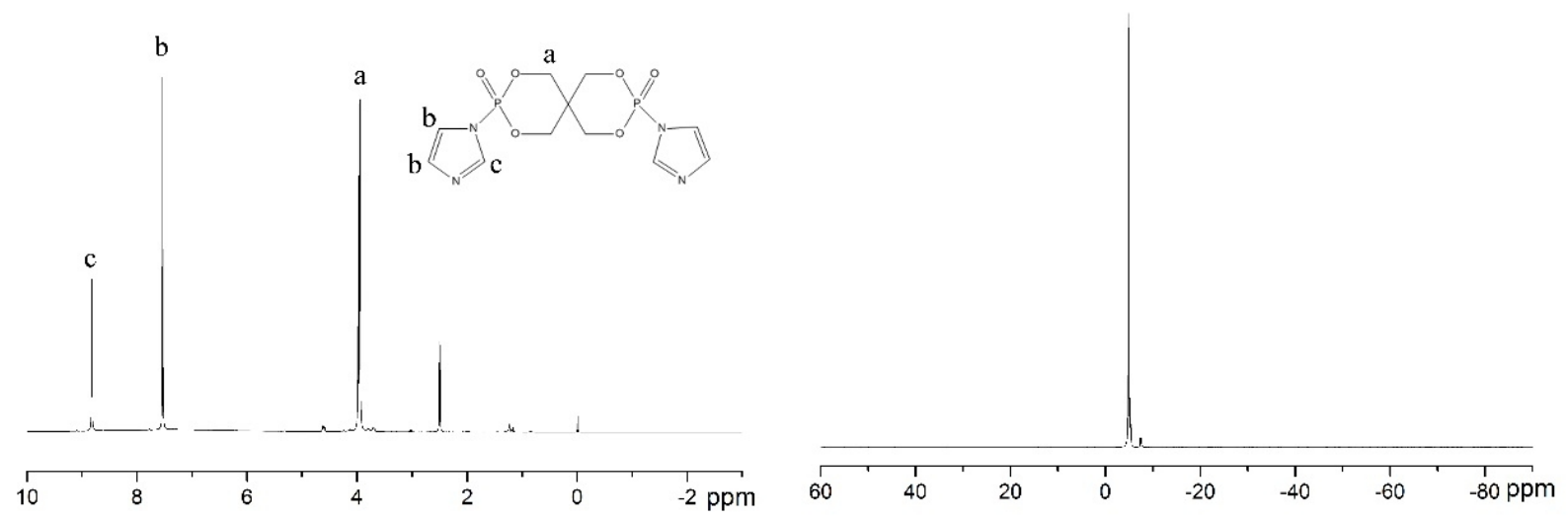

Fig. $2{ }^{1} \mathrm{H}$ NMR and ${ }^{31} \mathrm{P}$ NMR spectra of ISCP.

Fire Performance of EP Composites. ISCP has been designed as a novel intumescent flame retardant, which is expected to enhance flame retardancy to EP. LOI and UL-94 tests were employed to evaluate the flame retardancy of ISCP. It can be seen from Table 1, EP burnt easily, accompanied with dripping, and its LOI value was only 19.5 . The addition of ISCP improved the LOI value a lot compared with EP-0, the maximum LOI value reached 36.8 when ISCP was $20 \mathrm{wt} . \%$, an increase of $88.7 \%$ in comparison with that of EP-0. Also, from the UL-94 tests, EP-4 could achieve V-0 rating simultaneously. It clearly showed that ISCP had certain flame retardant efficency on EP.

Table 1 Formulas, flame retardancy of EP and EP/ISCP composites

\begin{tabular}{cccccc}
\hline Samples & EP [wt.\%] & ISCP [wt.\%] & LOI [\%] & UL-94 & Dripping \\
\hline EP-0 & 100 & 0 & 19.5 & Fail & Y \\
EP-1 & 95 & 5 & 24.6 & Fail & Y \\
EP-2 & 90 & 10 & 26.8 & V-1 & N \\
EP-3 & 85 & 15 & 30.8 & V-1 & N \\
EP-4 & 80 & 20 & 36.8 & V-0 & N \\
\hline
\end{tabular}

CONE Calorimeter Analysis. To further comparatively study the flame ratardancy of the EP composites in a real fire, CONE calorimeter was carried out to evaluate the combustion performance. The heat release rate (HRR) curves and total heat release (THR) curves measured by CONE calorimetry are illustrated in Fig. 3. It is found that EP-0 burnt fiercely after ignition and the peak HRR (PHRR) value was $898 \mathrm{~kW} / \mathrm{m}^{2}$. THR value of non-flame retarded EP was $60.8 \mathrm{MJ} / \mathrm{m}^{2}$. However, when 20 wt.\% ISCP was added, the EP/ISCP composite's PHRR and THR remarkably decreased. Its PHRR value dropped to $311 \mathrm{~kW} / \mathrm{m}^{2}$, a decrease of $65.4 \%$ in comparison with that of EP- 0 . The THR value of EP-4 significantly dropped to $27.6 \mathrm{MJ} / \mathrm{m}^{2}$, a decrease of $54.6 \%$ in comparison with that of EP-0. Direct observations revealed that the first PHRR was attributed to ISCP degradation and intumescent shield formation. In addition, the surface of EP-4 gradually produced the char layer and inhibited the heat from pyrolyzing the underlying substrate. However, the shield degraded sustainly when it faced massive heat and the residual materials degraded and formed a new intumescent shield as well. Consequently, combustion intensity was simultaneously enhanced and the second PHRR appeared, indicating that more compact and stable char layers were gradually generated on the surface of EP/ISCP composites [6]. According to the above results, it can be concluded that the incorporation of ISCP greatly enhanced the flame retardancy of EP. 

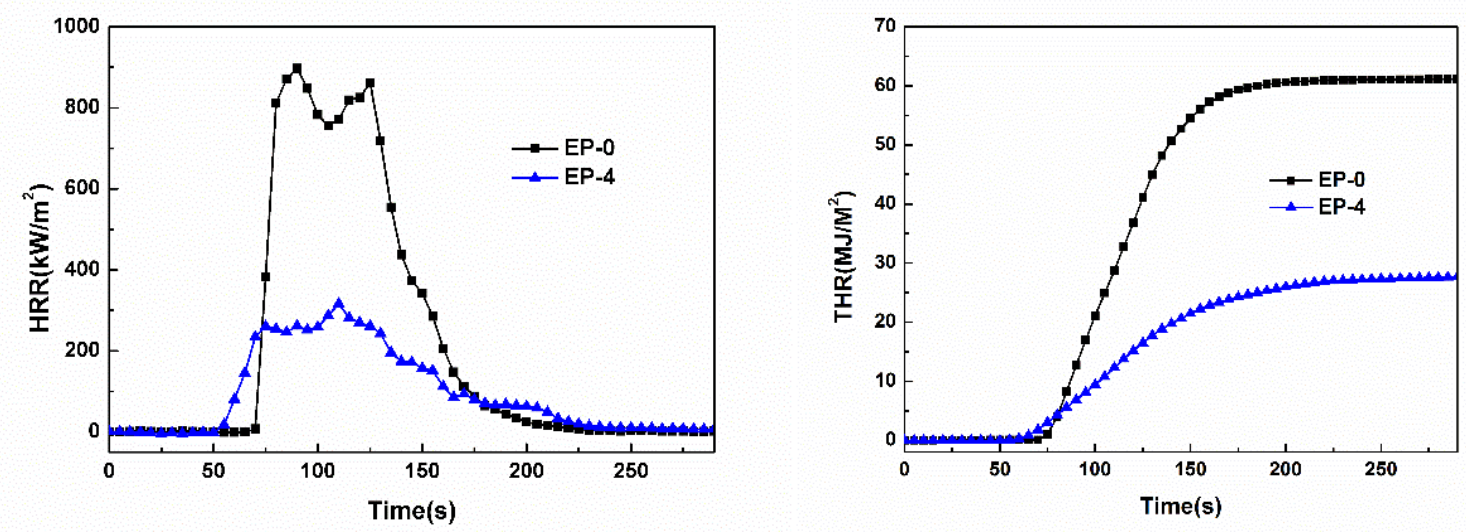

Fig. 3 HRR and THR curves from CONE tests of EP-0 and EP-4 composites.

Thermal Properties of EP/ISCP Composites. TGA and DTG curves of EP-0 and EP-4 are presented in Fig. 4. The results showed their thermal stabilities and thermal degradation behaviors. Both the specimens decomposed in one step, indicating that the addition of ISCP rarely changed the degradation mechanism of EP. The initial weight loss of EP-0 took place at $337{ }^{\circ} \mathrm{C}$, with a maximum weight loss rate at $369^{\circ} \mathrm{C}$, and just $8.1 \%$ residual char was left. Nevertheless, EP-4 started to decompose at $310{ }^{\circ} \mathrm{C}$, with a maximum weight loss rate at $351{ }^{\circ} \mathrm{C}$. The residual char from EP-4 at $800{ }^{\circ} \mathrm{C}$ was as high as $24.1 \%$, indicating higher thermal stability and good flame retardancy properties in condensed phase. The addition of ISCP decreased the initial degradation temperature, that's because P-O bonds of ISCP were prone to cracking at the heating process firstly, then phosphorus units removed from EP and volatilized into gaseous phase resulting in lower temperature. TGA tests revealed that during the combustion, ISCP could generate the phosphorus-containing radicals and inhibit the propagation of flame [7]. The elimination of non-flammable gas and the formation of continuous intumescent char could improve flame retardancy of EP.
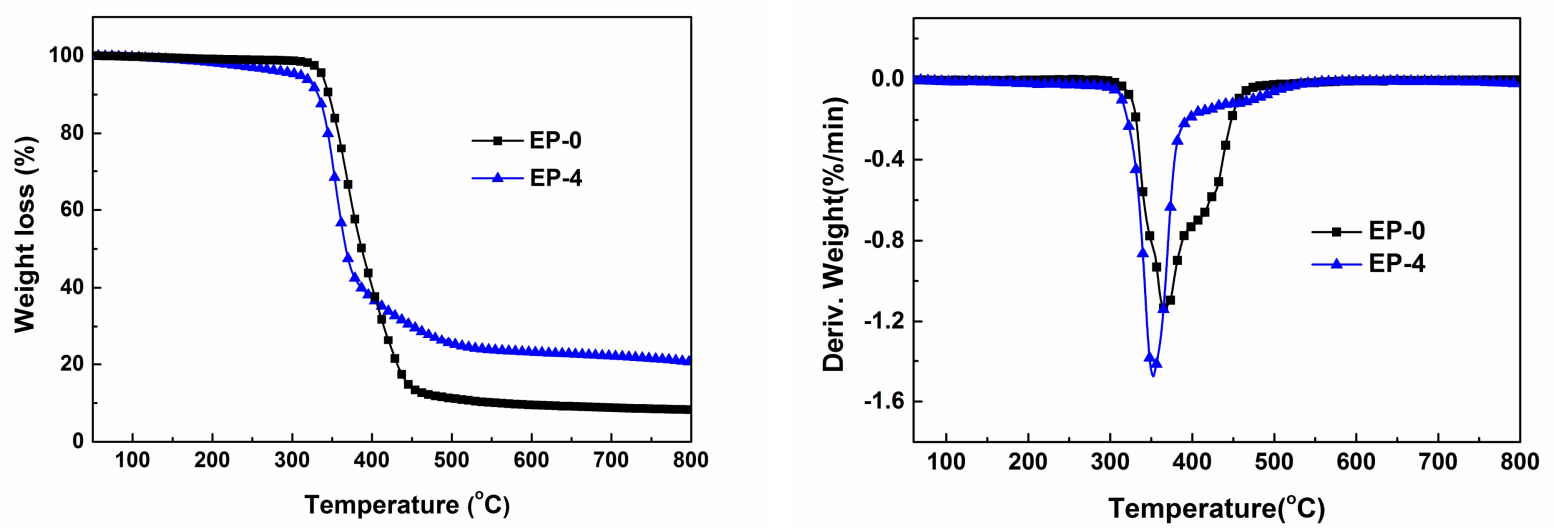

Fig. 4 TGA and DTG curves of EP-0 and EP-4 composites

Morphologies of the Residual Char. The residual char from EP-4 after CONE tests was studied by SEM. Fig. 5 shows micrograghs of the exterior and interior residual char. The exterior char was uniform and compact. The char layer could effectively slow down the heat and mass transfer, and providing better heat insulation in both gaseous and condensed phase.Furthermore, holes and cracks existed in the interior of residual char. During combustion, volatiles and bubbles could agitate the melted EP and cooperate with residual char formation [8]. Gaseous volatiles were also released, contributing to a foaming process during combustion. SEM morphologies indicated that ISCP had a certain intumescent effect. These results are in accordance with those of LOI and CONE tests. 


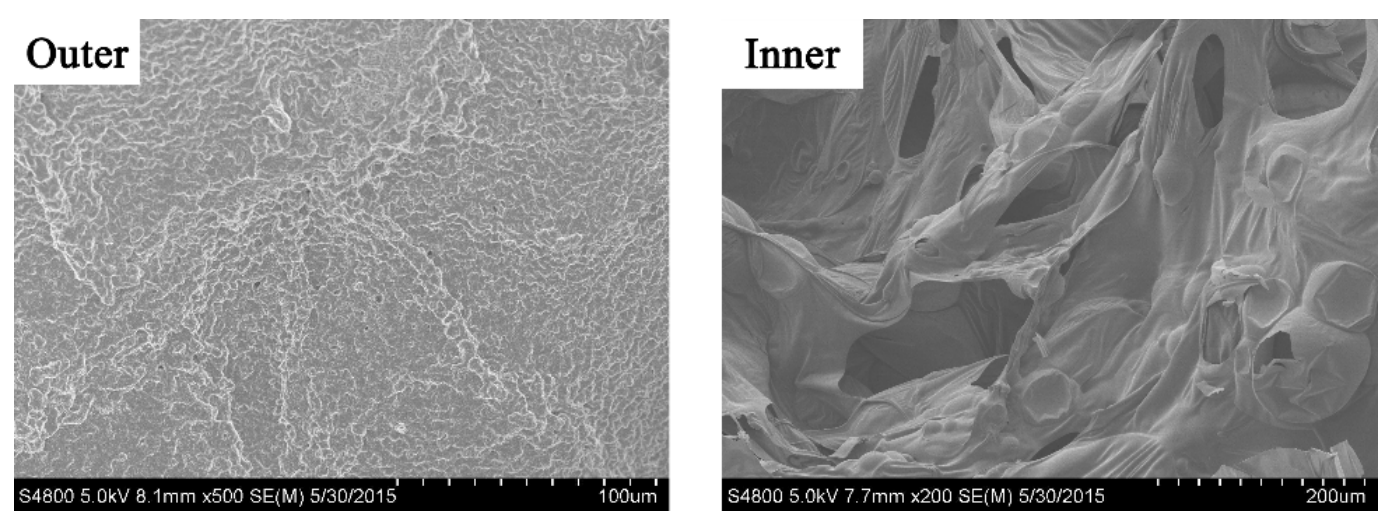

Fig. 5 SEM morphologies of the residual char of EP-4 from CONE tests.

\section{Conclusions}

An efficient spirocyclic halogen-free flame retardant ISCP was successfully synthesized and characterized. Results of LOI, UL-94, and CONE calorimetry tests demonstrated that the ISCP effectively enhanced flame retardancy of EP composites, which could improve the LOI value from 19.5 to 36.8, pass the UL-94 V-0 rating when $20 \mathrm{wt} . \%$ ISCP was added, and CONE tests showed that ISCP could decrease the PHRR and THR of EP composites remarkably. TGA tests revealed that ISCP could increase the amount of residual char and eliminate the mass of pyrolysis products during combustion. SEM morphologies indicated that ISCP greatly facilitated the char-forming process and generated uniform and compact intumescent char layer, leading to EP with improved flame retardancy. Consequently ISCP led to excellent intumescent flame retardant performance on EP.

\section{References}

[1] T. Mariappan, C.A. Wilkie, Flame retardant epoxy resin for electrical and electronic applications, Fire Mater. 38 (2014) 588-98.

[2] J. Sun, X.D. Wang, D.Z. Wu, Novel spirocyclic phosphazene-based epoxy resin for halogen-free fire resistance: synthesis, curing behaviors, and flammability characteristics, Appl. Mater. Interfaces. 4 (2012) 4047-61.

[3] C.S. Wang, J.Y. Shieh, Phosphorus-containing epoxy resin for an electronic application, J. Appl. Polym. Sci. 73 (1999) 353-61.

[4] W. Liu, L. Chen, Y.Z. Wang, A novel phosphorus-containing flame retardant for the formaldehyde-free treatment of cotton fabrics, Polym. Degrad. Stab. 97 (2012) 2487-91.

[5] L.S. Wang, Y. Liu, R. Wang, Solubilities of some phosphaspirocyclic compounds in selected solvents. J. Chem. Eng. Data. 51 (2006) 1686-89.

[6] P.A. Song, Z.P. Fang, L.F. Tong, Z.B. Xu, Synthesis of a novel oligomeric intumescent flame retardant and its application in polypropylene, Polym. Eng. Sci. 49 (2009) 1326-31.

[7] X.J. Lai, S. Tang, S.; H.Q. Li, X.R. Zeng, Flame-retardant mechanism of a novel polymeric intumescent flame retardant containing caged bicyclic phosphate for polypropylene, Polym. Degrad. Stab. 113 (2015) 22-31.

[8] W. Zhao, J.P. Liu, H. Peng, J.Y. Liao, X.J.Wang, Synthesis of a novel PEPA-substituted polyphosphoramide with high char residues and its performance as an intumescent flame retardant for epoxy resins, Polym. Degrad. Stab. 118 (2015) 120-29. 